\title{
Prognostic factors for patients with metastatic breast cancer: a literature review
}

\author{
Mengyu $\mathrm{Hu}^{1}$, Bin $\mathrm{Shao}^{2}$, Ran $\mathrm{Ran}^{2}$, Huiping $\mathrm{Li}^{2}$ \\ ${ }^{1}$ Radiation Oncology Center, Chongqing University Cancer Hospital, Chongqing, China; ${ }^{2}$ Key Laboratory of Carcinogenesis and Translational \\ Research (Ministry of Education/Beijing), Department of Breast Oncology, Peking University Cancer Hospital \& Institute, Beijing, China \\ Contributions: (I) Conception and design: H Li, M Hu; (II) Administrative support: B Shao; (III) Provision of study materials or patients: R Ran; (IV) \\ Collection and assembly of data: M Hu; (V) Data analysis and interpretation: M Hu, H Li; (VI) Manuscript writing: All authors; (VII) Final approval \\ of manuscript: All authors. \\ Correspondence to: Huiping Li, MD, PhD. Key Laboratory of Carcinogenesis and Translational Research (Ministry of Education/Beijing), \\ Department of Breast Oncology, Peking University Cancer Hospital \& Institute, No. 52, Fucheng Road, Haidian District, Beijing 100142, China. \\ Email: huipingli2012@hotmail.com.
}

Background: To treat metastatic breast cancer (MBC) more precisely, many efforts have been made to identify prognostic factors of $\mathrm{MBC}$ in many studies. This review aims to qualitatively summarize these studies and to provide a reference for the research of MBC.

Methods: Relevant papers were searched on PubMed, with the search terms including MBC, prognostic factors and prognosis, and the studies aimed at exploring prognostic factors for patients with histologically confirmed MBC, including stage IV at initial diagnosis and metastatic recurrence, were included.

Results: A total of 30 papers were included at last. An analysis of prognostic factors frome those studies was conducted. Age at primary diagnosis (6 studies), performance status (4 studies), histological grade (4 studies), hormonal receptor (HR) status (9 studies) and site of metastasis (12 studies) were universally acknowledged prognostic factors. There were four studies revealing that short DFS was significantly associated with better OS, while there was one study not revealing this association. There were various results in different studies with a reference to efficacy. Surgery and endocrine therapy were related to a better prognosis ( 3 studies). Targeted therapies could also conduce to the prognosis. However, there was still a contention on the role of radiotherapy. In particular, a model was brought out to calculate the risk of death in MBC. Meanwhile, it was found that some biomarkers are related to prognosis as well as per the latest findings in some studies.

Conclusions: In summary, intrinsic characteristics of tumors such as HR status and histological grade are the main factors affecting the prognosis of patients with MBC. Besides traditional factors, some new drugs and biomarkers are also associated with the prognosis of patients with MBC. In the future, the focus of studies shall be on the construction of a practical and high-quality model to predict the risk of death in MBC patients.

Keywords: Breast cancer (BC); metastasis; prognostic factor; review

Submitted May 18, 2020. Accepted for publication Mar 05, 2021.

doi: $10.21037 /$ tcr-20-2119

View this article at: http://dx.doi.org/10.21037/tcr-20-2119

\section{Introduction}

Breast cancer (BC) is one of the most common malignant tumors and the leading cause of cancer death among women $(1,2)$. Metastasis is the main reason causing death in patients with BC (3). Metastasis would occur in approximately $5 \%$ to $10 \%$ of patients with BC. Besides, about $30 \%$ of women will finally develop relapsed $\mathrm{BC}$ after the initial diagnosis at early stages $(4,5)$. Despite of medical advances, the 5-year survival rate of patients with metastatic breast cancer (MBC) 
is still less than $30 \%$ (6).

In order to treat $\mathrm{MBC}$ more precisely, many efforts have been made to identify prognostic factors of $\mathrm{MBC}$ in many studies. It has been found in some studies that patient characteristics such as age and performance status are associated with the prognosis of patients with $\mathrm{MBC}$ $(1,7)$. Moreover, it has been found in other studies that characteristics of tumors such as molecular type and histological grade are related to the survival of these patients $(8,9)$. According to molecular type and other prognostic factors, specific treatment strategies, such as anti-HER2 therapy and endocrine therapy, could be adopted to improve the outcome of patients.

Due to the fact that the prognosis of $\mathrm{MBC}$ is affected by various factors, it is significant to better evaluate the prognosis of individual patients through combining those factors together. There are various results on prognostic factors of $\mathrm{MBC}$ from different studies involving different subtypes. This review aims to make a summarization of these studies and give some suggestions on future studies, so that researchers could easily find the specific prognostic factors and relevant information about different subtypes of $\mathrm{MBC}$, thus providing a reference for the research of MBC. We present the following article in accordance with the PRISMA reporting checklist (available at http://dx.doi. org/10.21037/tcr-20-2119).

\section{Methods}

\section{Literature search and selection criteria}

Relevant papers were searched on PubMed, with the search terms including MBC (title), prognostic factors (title) and prognosis (title). Abstracts from the selected papers were reviewed by an assessor at first. Then the full papers were further evaluated according to the selection criteria by another assessor. The studies included should aim at exploring prognostic factors for patients with histologically confirmed MBC, including stage IV at the initial diagnosis and metastatic recurrence. The studies were excluded in case of any of the following criteria was met: (I) the publication type was comment, letter or review; (II) there was any other malignant tumor with the patients; (III) the paper was published before 1990 .

\section{Data extraction and analysis}

Data extraction was performed independently by two assessors. Information on patient selection, sample size and endpoints were extracted for each study (Table 1). Prognostic factors of MBC in each study were summarized (Table 2 and Figure 1) and analyzed. Results from different subtypes of BC were demonstrated and discussed separately.

The endpoints included overall survival (OS), breast cancer specific survival (BCSS), disease-free survival (DFS), or progression-free survival (PFS) in these studies. OS was defined as the time from the diagnosis to death or the last visit for patients presenting primary metastatic breast cancer (PMBC) and the time from the metastasis to death or the last visit for patients presenting $\mathrm{BC}$ metastatic recurrence. BCSS was defined as the BC related survival time. DFS was defined as the time from the diagnosis of $\mathrm{BC}$ to the metastatic recurrence. PFS was defined as the disease progression after the diagnosis of $\mathrm{BC}$ metastatic recurrence.

\section{Results}

\section{Selection and characteristics of studies}

The search strategy identified 113 English-language papers in total. After the screening of the title and abstract, a total of 69 papers were selected. Throug the text review, 39 papers were further excluded. Finally, 30 papers were determined as per the inclusion criteria.

These 30 included studies were published between 1998 and 2019, in which Kaplan-Meier method and Cox proportional hazard regression model were adopted to analyze the effect of different clinicopathological characteristics on the prognosis of patients diagnosed with MBC (Table 1). There were 7 studies including patients diagnosed with either the $\mathrm{PMBC}$ or initial stage III subsequently developing metastatic disease (8,10-15). Among them, there was 1 study identifying patients diagnosed with bone as only metastasis site (13) and were 2 studies focusing on human epidermal growth factor receptor 2 oncogene (HER2) positive patients $(11,14)$. There were 10 studies focusing on BC patients with metastatic recurrence $(1,2,7,9,16-21)$, in which there was 1 study focusing on bone metastasis (BM) (16), 1 study focusing on central nervous system (CNS) metastasis (17), 1 study focusing on brain metastasis (20) and 1 study focusing on patients older than 70 years old, respectively (19). There were 3 studies including patients diagnosed with $\mathrm{PMBC}$ (22-24), in which there was 1 study focusing on hormone receptor (HR) positive patients. There were 10 studies focusing on the effect of specific factors, such as drugs and 


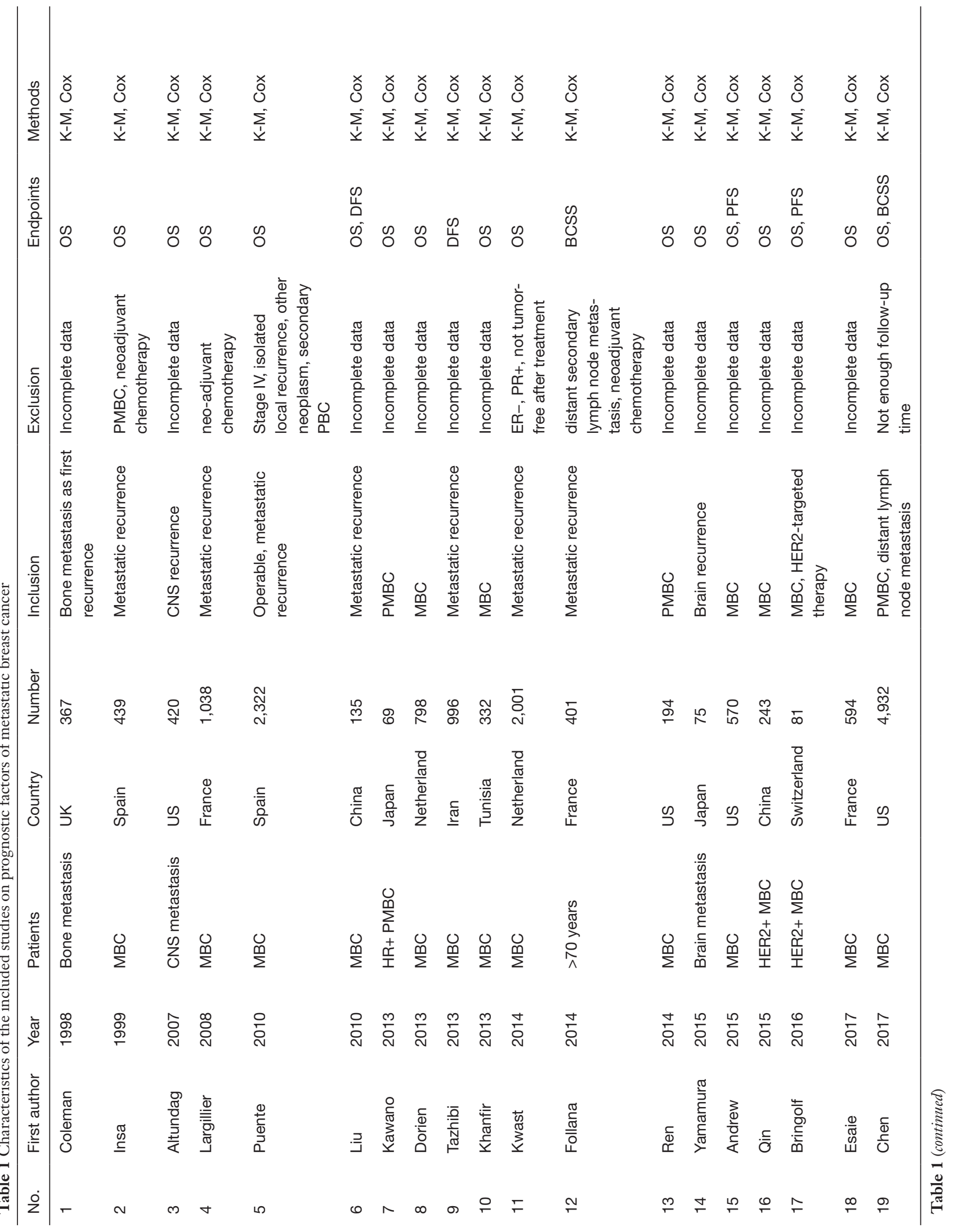




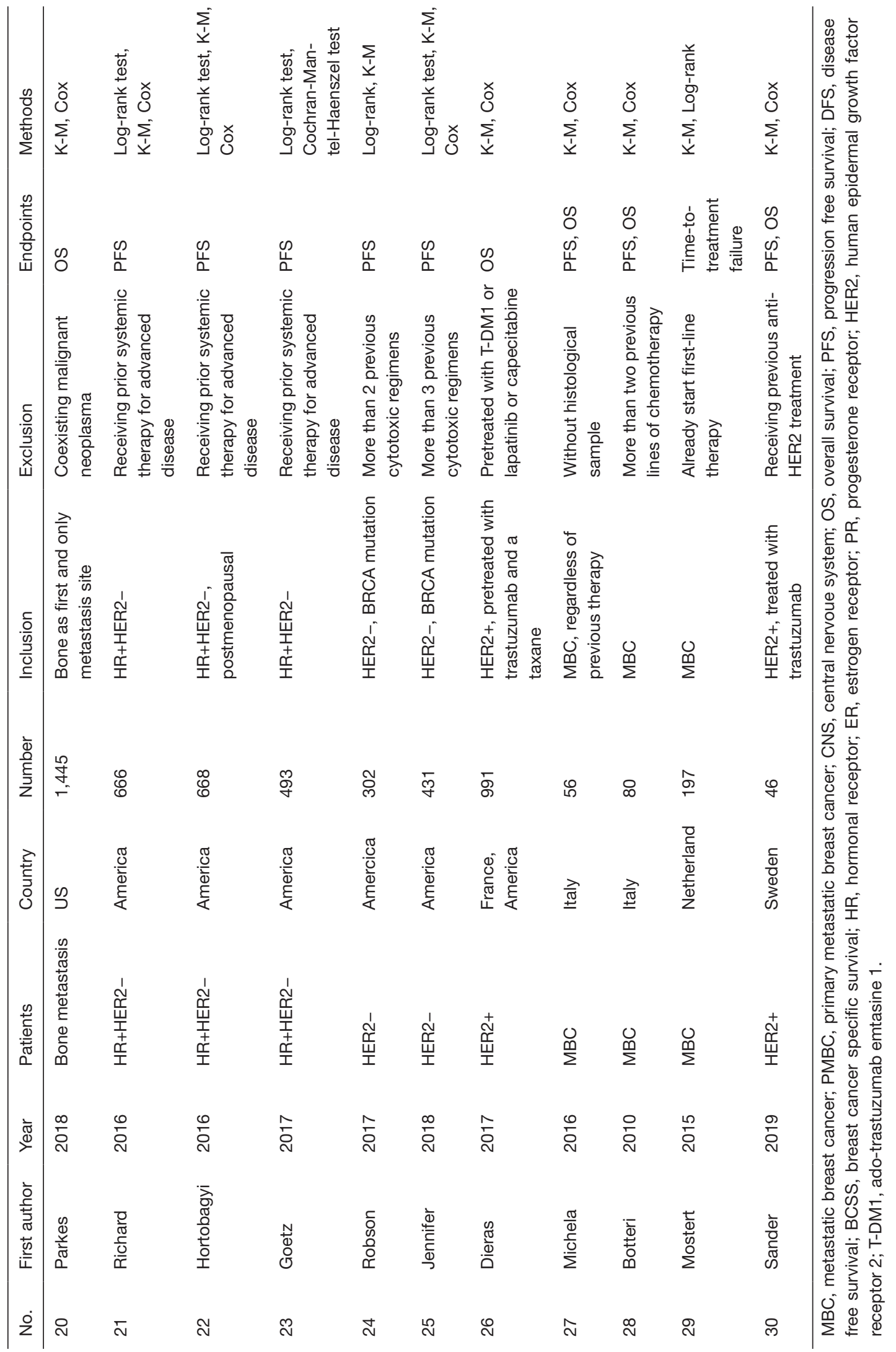


Table 2 Prognostic factors of metastatic breast cancer in each study

\begin{tabular}{|c|c|c|c|c|c|}
\hline No. & First author & Year & Patients & Number & Prognostic factors \\
\hline 3 & Altundag & 2007 & CNS metastasis & 420 & Age at diagnosis, ER \\
\hline 4 & Largillier & 2008 & MBC & 1,038 & $\begin{array}{l}\text { Age at diagnosis, HR, site of metastasis, primary tumor size, } \\
\text { histological grade, adjuvant chemotherapy }\end{array}$ \\
\hline 5 & Puente & 2010 & $\mathrm{MBC}$ & 2,322 & $\begin{array}{l}\text { Age at diagnosis, HR, lymph node, site of metastasis, chemotherapy, } \\
\text { number of hormonal therapy in metastasis, response to first line } \\
\text { therapy }\end{array}$ \\
\hline 6 & Liu & 2010 & $\mathrm{MBC}$ & 135 & Performance status, chemotherapy \\
\hline 7 & Kawano & 2013 & $\mathrm{HR}+\mathrm{PMBC}$ & 69 & PR, response to first/second endocrine therapy \\
\hline 11 & Kwast & 2014 & MBC & 2,001 & $\begin{array}{l}\text { Age at metastatic diagnosis, HR, histological grade, Her } 2 \text {, DFS, site } \\
\text { of metastasis, surgery, chemotherapy, endocrine treatment }\end{array}$ \\
\hline 12 & Follana & 2014 & $>70$ years & 401 & HR, lymph node, site of metastasis, DFS \\
\hline 13 & Ren & 2014 & $\mathrm{MBC}$ & 194 & Race, ER \\
\hline 14 & Yamamura & 2015 & Brain metastasis & 75 & Molecular subtype, performance status, single brain metastatic tumor \\
\hline 15 & Andrew & 2015 & MBC & 570 & $\begin{array}{l}\text { Race, BMI, stage, molecular subtype, site of metastasis, adjuvant } \\
\text { hormones, local therapy, adjuvant radiotherapy }\end{array}$ \\
\hline 16 & Qin & 2015 & HER2+ MBC & 243 & $\begin{array}{l}\text { Surgery, endocrine therapy, anti-Her2 therapy, performance status, } \\
\text { brain metastasis }\end{array}$ \\
\hline 20 & Parkes & 2018 & Bone metastasis & 1,445 & $\begin{array}{l}\text { Multiple bone metastasis, both axial and appendicular skeleton } \\
\text { metastasis }\end{array}$ \\
\hline 21 & Richard & 2016 & HR+HER2- & 666 & Palbociclib combined with letrozole (vs. Al) \\
\hline 22 & Hortobagyi & 2016 & HR+HER2- & 668 & Ribociclib combined with letrozole (vs. Al) \\
\hline 23 & Goetz & 2017 & $\mathrm{HR}+\mathrm{HER} 2-$ & 493 & Abemaciclib combined with $\mathrm{Al}$ (vs. Al) \\
\hline 24 & Robson & 2017 & HER2- & 302 & Olaparib (vs. single chemotherapy) \\
\hline 25 & Jennifer & 2018 & HER2- & 431 & Talazoparib (vs. single chemotherapy) \\
\hline 26 & Dieras & 2017 & HER2+ & 991 & T-DM1 (vs. lapatinib plus capecitabine) \\
\hline 27 & Michela & 2016 & MBC & 56 & Circulating tumor cell \\
\hline 28 & Botteri & 2010 & $\mathrm{MBC}$ & 80 & Circulating tumor cell \\
\hline 29 & Mostert & 2015 & $\mathrm{MBC}$ & 197 & Circulating tumor cell \\
\hline 30 & Sander & 2019 & HER2+ & 46 & ERBB2 and PTPN2 gene copy numbers \\
\hline
\end{tabular}

MBC, metastatic breast cancer; PMBC, primary metastatic breast cancer; CNS, central nervoue system; DFS, disease free survival; HR, hormonal receptor; ER, estrogen receptor; PR, progesterone receptor; HER2, human epidermal growth factor receptor 2; T-DM1, adotrastuzumab emtasine 1; BMI, body mass index; Al, Aromatase inhibitor. 
Age at diagnosis

Age at metastasis

Performance status

BMI

Race

Histological grade

Molecular subtype

ER/PR

HER2

T stage

Tumor size

Lymph node status

Site of metastasis

DFS

surgery

Endocrine therapy

Chemotherapy

Anti-HER2 therapy

Response to first line therapy

Response to first/second endocrine therapy

Radiotherapy

CDK4/6

PARP inhibitor

TDM-1

Circulating tumor cell

ERBB2 \& PTPN2 gene copy

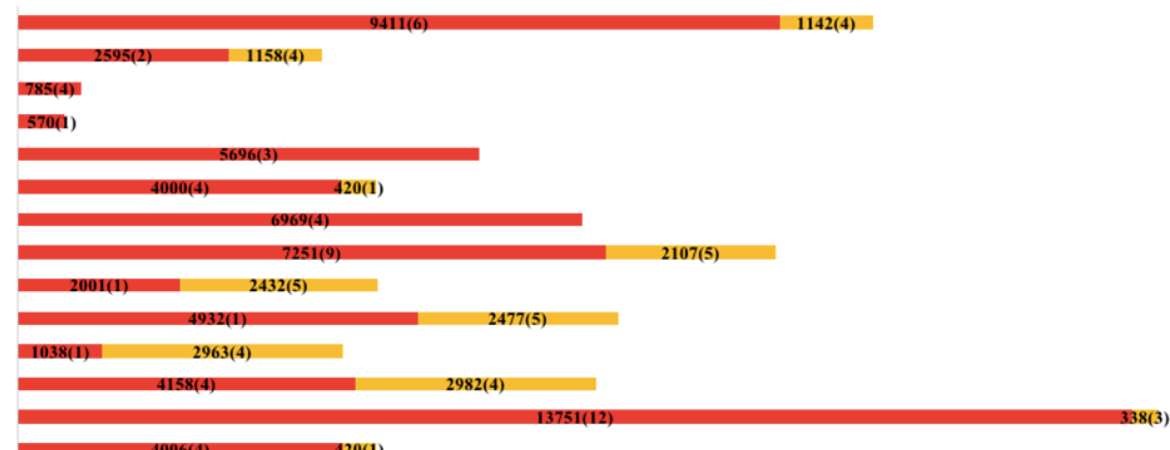

4006(4) $420(1)$
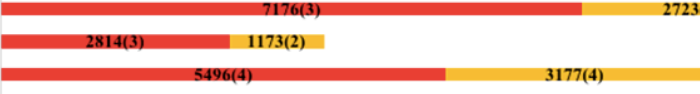

243(1)

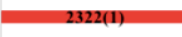

169(1)

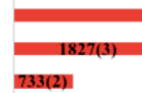

\section{3(2)}

991(1)

333(3) Number of patients (number of studies)

46(1)

2000

4000

6000

8000

10000

12000

14000

16000

Figure 1 Prognostic factors of MBC identified in at least one study. BMI, body mass index; ER, estrogen receptor; PR, progesterone receptor; DFS, disease free survival; HER2, human epidermal growth factor receptor 2; CDK4/6, cyclin-dependent kinase 4/6; PARP, poly (adenosine diphosphate-ribose) polymerase; T-DM1, ado-trastuzumab emtasine 1; MBC, metastatic breast cancer.

biomarkers, on prognosis (25-34).

\section{Study results}

\section{Prognostic factors of MBC, including PMBC and metastatic recurrence}

Khanfir et al. (15) analyzed 332 patients with histologically confirmed PMBC and metastatic recurrence BC in Tunisia. They found that good performance status (PS), non-visceral metastatic recurrence and $\leq 70$ years old were related to better OS (Table 2). Marshall et al. (12) conducted research into 594 patients from the only registry specialized BC center in France. The results showed that molecular subtypes, histological grade, metastasis site and age at diagnosis were independent prognostic factors of the OS in MBC, while there was no significant difference in OS between patients with $\mathrm{PMBC}$ and metastatic recurrence. Lobbezoo et al. (8) conducted a research into 798 patients in eight hospitals in the Netherlands. They divided the patients into four groups according to HR and HER2 status of primary tumor or metastatic lesion, and found that patients with the HR+/HER2 + subtype had better OS than the patients with other subtypes. Meanwhile, it was found that $\leq 50$ years old at primary diagnosis, DFS $\geq 24$ months, adjuvant endocrine therapy, and absence of visceral, brain and multiple metastases were favorable prognostic factors. In another study, Bishop et al. (10) identified $570 \mathrm{MBC}$ patients treated at MD Anderson. They defined a complete response according to RECIST criteria as no-evidence-ofdisease (NED). They found attaining NED status was not related to OS, but it would influence the survival at 2 and 3 years.

\section{Patients with $\mathrm{BC}$ metastatic recurrence}

Largillier et al. (18) reported a study of 1038 patients diagnosed with metastatic recurrence and not receiving adjuvant chemotherapy in France. They found that age, size of primary tumor, histological grade, HR status, metastasis site and adjuvant chemotherapy were independent prognostic factors of MBC, while DFS was not significantly associated with OS. Similarly, in other three studies $(2,7,21)$, researchers identified the prognostic factors of patients with metastatic recurrence in Spain, China and Iran respectively. However, in the study from Spain, DFS remained 
independently associated with OS after the first recurrence. In the study from China, it was found that PS was related to OS besides the mentioned factors, while there was no such relation for DFS. There was another study evaluating patients in Spain (1), which included more population compared with the study in Spain. This study collected the clinical data of 2,322 patients from 50 hospitals. In this study, it was found that age, tumor characteristics and treatment were related to prognosis, but DFS appeared not to be associated with prognosis as per the multivariate analysis.

\section{Patients with PMBC}

Ren et al. (23) identified 194 patients with distant metastasis at the time of diagnosis in the United States. They found race and HR status could exert impact on OS. In another study from the United States (24), researchers aimed to identify clinical characteristics related to distant metastasis by age groups in a large population. They found that age, HR and other factors such as T stage and the site of metastasis, could affect OS and BCSS of these patients. In a study from Japan, Kawano et al. (22) conducted an analysis of $69 \mathrm{HR}$ positive PMBC patients. They found that progesterone receptor status and clinical benefit rate from the first-line endocrine therapy were independent prognostic factors of OS.

\section{Prognostic factors of $\mathrm{MBC}$ with bone metastasis}

There were two studies discovering the factors affecting prognosis of MBC patients with BM. Coleman et al. (16) identified 367 patients with the first recurrence site in bone. They found that age at diagnosis, histology, HR status, DFS, bone disease at presentation would affect the prognosis. From another point of view, Parkes et al. (13) described the association among bone pain, location, number, type of $\mathrm{BM}$ and OS in MBC patients with BM. They conducted an analysis of 1445 patients with bone as the only metastatic site, and found that multiple bone metastases and both appendicular and axial skeleton metastases were risk factors for decreased OS in these patients. Patients with both appendicular and axial metastases had a $69 \%$ (hazard ratio, $1.69 ; 95 \%$ CI, $1.31-2.19$ ) and $65 \%$ (hazard ratio, $1.65 ; 95 \%$ CI, 1.26-2.17) of increased hazard of death compared to patients with metastasis confined to appendicular and axial skeleton respectively.

\section{Prognostic factors of $\mathrm{MBC}$ with CNS metastasis}

Altundag et al. (17) retrospectively evaluated data from 420 patients with $\mathrm{BC}$ and CNS metastasis. CNS metastasis was defined as a metastasis in the brain and/or leptomeningeal disease. The results showed that patients with ER positive (hazard ratio, 0.69; 95\% CI, 0.55-0.87) and younger age (hazard ratio, 1.01; 95\% CI, 1-1.02) had better outcome, while other factors, such as PR status, histological grade, tumor size and lymph node classification, were not significantly associated with the survival. In this study, there were only 248 patients with known information of HER2 status. Patients with HER2 positive lived longer than those with negative HER2 (median time 11 vs. 6 months). Yamamura et al. (20) conducted research into 75 early BC patients subsequently developing brain metastasis. The results indicated that luminal HER2 cancer, favorable PS and single metastatic brain tumor independently affected the prognosis of those patients.

\section{Prognostic factors of MBC with positive HER2}

Qin et al. (11) assessed $243 \mathrm{MBC}$ patients with HER2 positive in southern China. They found that anti-HER2 therapy (trastuzumab or lapatinib), endocrine therapy and surgical intervention were favorable independent prognostic factors for OS in these patients, while poor PS and brain metastasis were unfavorable factors. In another study, Bringolf et al. (14) identified 81 patients with HER2 positive, among whom there were 25 receiving HER2targeted therapy in Switzerland. They found that only primary brain metastasis was an unfavorable prognostic factor, while other factors were not significantly associated with the prognosis of these patients. Median OS of patients with primary brain metastasis was 1.9 years (95\% CI, 1.7-2.2 years), while median OS of all patients with brain metastasis was 26 months (95\% CI, 19.9-32.0 months).

\section{Latest findings on prognostic factors of MBC}

Besides the mentioned traditional clinicopathological factors, the effects of new drugs and biomarkers on the prognosis of MBC patients were explored in some studies. Cyclin - dependent kinase 4/6 (CDK 4/6) inhibitor is a newly developed drug to overcome the intrinsic or acquired resistance to endocrine therapy in patients with HR positive/HER2 negative patients (31). Finn et al. (25) found that in untreated patients with HR positive/HER2 negative patients, palbociclib combined with letrozole would result in a significantly longer PFS than that with letrozole alone (24.8 vs. 14.5 months). In other two clinical trials, it was also demonstrated that drugs targeting CDK4/6 combined with aromatase inhibitor (AI) could 
improve PFS compared to that with AI alone (26,35). Recently, the role of poly (adenosine diphosphate-ribose) polymerase (PARP) inhibitor in HER2 negative especially triple negative MBC has been identified. Robson et al. (28) found that among HER2 negative patients with BRCA mutation, the median PFS of patients treated with Olapalib was 2.8 months, which was longer than that with standard single agent chemotherapy. Similarly, it was demonstrated that talazoparib, another PARP inhibitor, could provide more significant benefits in terms of PFS than standard chemotherapy in another phase III clinical trial (30). Adotrastuzumab emtasine (T-DM1) is a compound consisting of anti-HER2 drug trastuzumab and cytotoxic emtasine, which provides a treatment for patients who have received trastuzumab for $\mathrm{MBC}$ or have disease progression after adjuvant trastuzumab therapy (29). In a phase III clinical trial, Diéras et al. conducted research into HER2 positive MBC patients who have been pretreated with trastuzumab and a taxane, and they found that median OS was longer with T-DM1 than with lapatinib plus capecitabine (29.9 vs. 25.9 months) (27).

Circulating tumor cells (CTC) are tumor cells shed in blood from primary tumors and might represent the risk of metastasis. Fehm et al. found PFS and OS would decreased as the increase of CTC at baseline (36). Bulfoni et al. found CD45 negative CTC co-expressing epithelial and mesenchymal markers were significantly related to poor PFS and OS (37). In another study, it was demonstrated that a 16 -gene CTC profile had a better prognostic performance than CTC count in MBC patients (38).

In patients treated with anti-HER2 therapy, trastuzumab resistance is a frequent challenge while there is relapsing or metastatic disease. HER2-associated PI3K/Akt signalling pathway may play a critical role in trastuzumab resistance. In a recent study, Ellegard et al. discovered that high-grade ERBB2 gene amplification level of more than 6 copies could improve OS and PFS, and more than 3 copies of PTPN2 gene were related to shorter OS and PFS in HER2 positive patients who received trastuzumab for treatment (32).

\section{Discussion}

In this review, an exploration on the prognostic factors of MBC from 30 studies has been carried out from different aspects. As per the results, it can be seen that age at primary diagnosis, tumor size, histological grade, HR status, HER2 status, lymph node involvement, site and number of metastasis are universally acknowledged prognostic factors (Figure 1), which reflects that the intrinsic characteristics of tumor are main factors affecting the prognosis of patients with MBC.

In previous studies, DFS was considered as a strong prognostic factor of MBC (39-41). In this study, however, there is still a contention on the relationship between DFS and OS. It was revealed in some studies that short DFS was significantly associated with worse OS $(2,8,9,16,19)$; however, there were some studies not revealing this association $(1,7,18)$. There are several possible reasons for those studies not showing the significant association between DFS and OS. Firstly, as per the study of Largillier et al. (18), DFS appears to be related to OS in the univariate analysis, but other parameters like tumor size are more powerful in the prediction of the specific survival in multivariate Cox model, which would eliminate the efficacy of DFS. Secondly, as per the study of Liu et al. (7), a prognostic tool named Nottingham Prognostic Index, along with DFS and other factors, has been included in the analysis. The Nottingham Prognostic Index has been constructed via histological grade, ER status, site of metastasis and DFS (42) and DFS indeed could exert an impact on this index. Finally, all the mentioned factors do not show a significant influence on OS except for Nottingham Prognostic Index. Since there is a relationship between Nottingham Prognostic Index and other factors, the results may not be persuasive enough. As we know, DFS reflects the aggressiveness of tumor. If there is a relationship between DFS and OS, DFS would be a useful factor to predict the survival time of patients and maybe a more powerful ajuvant treatment should be applied to patients to prolong the duration of DFS. Of course, a further investigation shall be made in the future.

Owing to advances in early detection and modern systemic therapy, the survival of patients with $\mathrm{MBC}$ improves over time (43) and the risk of death decreases by $1 \%$ in each year (44). It is suggested that the improvement in survival is related to treatment as per the results from a large multicenter study (43). Referring to the efficacy, there are various outcomes in different studies in this review. It could be found in some studies that adjuvant therapies, including chemotherapy, endocrine therapy, targeted therapy and radiotherapy, could prolong the survival time of patients with MBC, while there still are some studies not revealing the significant benefits on survival from systemic treatment $(1,2,7,8,18-21,24)$. However, due to the fact that most studies included are single-center studies, it is 
inevitable for the existence of patient selection bias, which would disturb the results. Nevertheless, in this review, there are two studies carried out in eight and fifty hospitals respectively $(1,8)$. As per the results from these two studies, the adjuvant treatment can be regarded as a favorable prognostic factor of $\mathrm{MBC}$. These results are relatively more convincing. A prospective study should be conducted to testify the impact of adjuvant therapy on survival.

Bone is the most common site of metastasis in BC. It is suggested that about $70 \%$ of patients with $\mathrm{BC}$ will develop BM (45). Bone metastasis are mainly distributed to axial skeleton, due to the possible fact that malignant cells could migrate through the axial bone marrow circulation which is connected with low-pressure valveless vertebral-venous plexus (16). As per the study of Parkes et al., 36\% of patients would have BM in the axial skeleton, $11 \%$ of patients would have $\mathrm{BM}$ in the appendicular skeleton, and $54 \%$ of patients would have BM in both the axial and appendicular skeleton. These results could confirm the theory mentioned above. In this study, metastasis in both axial and appendicular skeleton would result in worse outcome than in either axial or appendicular skeleton, which could help us to stratify the risk of death within patients diagnosed with BM and provide them with a more appropriate treatment.

CNS is one of the most common sites of metastasis and accounts for about $30 \%$ of all MBC (46). It has been assumed that the incidence of CNS metastasis would be higher in young, ER negative (47) and HER2 positive patients (48). As per the study of Altundag et al. (17), there is a better prognosis of $\mathrm{MBC}$ with CNS metastasis for young and ER positive patients. Although young patients are more likely to develop CNS metastasis, they tend to have better outcomes than old patients. However, for patients with ER negative, the prognosis is not optimistic due to both higher risk of developing CNS metastasis and poor prognosis after CNS metastasis. It has also been found by the authors that patients with HER2 positive tend to live longer than those with HER2 negative, which is consistent with the results from Yamamura et al. (20). Anti-HER2 therapy may be the main factor that could improve the prognosis of patients with HER2 positive.

HER2 proto-oncogene amplification can be found in about $25-30 \%$ of patients with BC (49) and has been thought to be related with poor prognosis in patients with BC. The treatment with anti-HER2 therapy dramatically alters the role of HER2 and improves the outcomes of patients with HER2-positive BC, which is even better than those with HER2-negative BC $(34,50,51)$. In this review, Qin et al. (11) has found that anti-HER2 treatment, such as trastzumab and lapatinib, could increase survival time of patients with HER2 positive, which is in line with the results from previous studies. As per another study by Bringolf et al. (14), it has been found that primary brain metastasis could exert impacts on the prognosis of patients with HER2 positive. Therefore, brain metastasis may be a useful prognostic factor in HER2 positive MBC in antiHER2-therapy era. However, there are certain limitations in this study. Owing to the fact that only 6 patients have been diagnosed with primary brain metastasis and 34 patients with secondary brain metastasis, the statistical comparison between the primary and secondary brain metastasis has been made indirectly in this review. Therefore, their results are not statistically significant and not persuasive enough.

It would be beneficial to identify factors affecting prognosis for clinical management strategies. Furthermore, it would conduce to guiding the treatment and followup more precisely via constructing a practical prognostic index. In the previous studies, there is one study proposing an index to calculate the risk of death in MBC (1). The researchers have collected data of patients with MBC from fifty hospitals. Through Cox proportional hazards model, a prognostic index has been constructed. 962 patients with complete data of all prognostic variables have been divided into the high, intermediate and low risk groups based on the index score. Finally, cumulative survival rate of each group has been calculated to validate the index. In this study, a useful tool has been provided to identify MBC patients with high risk of death who need more aggressive treatment. However, there are certain limitations in this study. First and most importantly, the qualification of a predictive model depends on the area under the ROC curve (AUC). The AUC value in this study is only 0.69 , which is not enough to distinguish patients with absolutely high risk. Second, the data are collected from the same database to validate the index in this study. It would be more convincing if the data can be collected from another database to validate the index. Despite all limitations, it is extremely meaningful to make an attempt to construct a practical model with the aim of predicting the risk of death in patients with MBC.

\section{Conclusions}

In this review, some prognostic factors of $\mathrm{MBC}$ have been discussed from different aspects. In summary, such 
intrinsic characteristics of tumors as tumor size and histological grade are main factors affecting the prognosis of patients with MBC. However, there is still a contention on the relationship between DFS and OS, which shall be investigated in the future. Stratified by molecular type and metastatic site, there are specific prognostic factors for different types of MBC. Besides traditional factors, some new drugs and biomarkers are also associated with the prognosis of patients with MBC. It would be beneficial to identify factors affecting prognosis for developing clinical management strategies, and furthermore, it would conduce to guiding the treatment more precisely via constructing a practical prognostic index. In the future, the research focus would be on the construction of a practical and highquality model with the aim of predicting the risk of death in patients with MBC.

\section{Acknowledgments}

The language of this paper is edited by an English professional, Kaige Shen.

Funding: None.

\section{Footnote}

Reporting Checklist: The authors have completed the PRISMA reporting checklist. Available at http://dx.doi. org/10.21037/tcr-20-2119

Conflicts of Interest: All authors have completed the ICMJE uniform disclosure form (available at http://dx.doi. org/10.21037/tcr-20-2119). The authors have no conflicts of interest to declare.

Ethical Statement: The authors are accountable for all aspects of the work in ensuring that questions related to the accuracy or integrity of any part of the work are appropriately investigated and resolved.

Open Access Statement: This is an Open Access article distributed in accordance with the Creative Commons Attribution-NonCommercial-NoDerivs 4.0 International License (CC BY-NC-ND 4.0), which permits the noncommercial replication and distribution of the article with the strict proviso that no changes or edits are made and the original work is properly cited (including links to both the formal publication through the relevant DOI and the license).
See: https://creativecommons.org/licenses/by-nc-nd/4.0/.

\section{References}

1. Puente J, Lopez-Tarruella S, Ruiz A, et al. Practical prognostic index for patients with metastatic recurrent breast cancer: retrospective analysis of 2,322 patients from the GEICAM Spanish El Alamo Register. Breast Cancer Res Treat 2010;122:591-600.

2. Insa A, Lluch A, Prosper F, et al. Prognostic factors predicting survival from first recurrence in patients with metastatic breast cancer: analysis of 439 patients. Breast Cancer Res Treat 1999;56:67-78.

3. Siegel RL, Miller KD, Jemal A. Cancer statistics, 2015. CA Cancer J Clin 2015;65:5-29.

4. Ruiterkamp J, Ernst MF, de Munck L, et al. Improved survival of patients with primary distant metastatic breast cancer in the period of 1995-2008. A nationwide population-based study in the Netherlands. Breast Cancer Res Treat 2011;128:495-503.

5. Dawood S, Broglio K, Ensor J, et al. Survival differences among women with de novo stage IV and relapsed breast cancer. Ann Oncol 2010;21:2169-74.

6. Brenner H, Gondos A, Arndt V. Recent major progress in long-term cancer patient survival disclosed by modeled period analysis. J Clin Oncol 2007;25:3274-80.

7. Liu MT, Huang WT, Wang AY, et al. Prediction of outcome of patients with metastatic breast cancer: evaluation with prognostic factors and Nottingham prognostic index. Support Care Cancer 2010;18:1553-64.

8. Lobbezoo DJ, van Kampen RJ, Voogd AC, et al. Prognosis of metastatic breast cancer subtypes: the hormone receptor/HER2-positive subtype is associated with the most favorable outcome. Breast Cancer Res Treat 2013;141:507-14.

9. Kwast AB, Voogd AC, Menke-Pluijmers MB, et al. Prognostic factors for survival in metastatic breast cancer by hormone receptor status. Breast Cancer Res Treat 2014;145:503-11.

10. Bishop AJ, Ensor J, Moulder SL, et al. Prognosis for patients with metastatic breast cancer who achieve a noevidence-of-disease status after systemic or local therapy. Cancer 2015;121:4324-32.

11. Qin T, Yuan ZY, Peng RJ, et al. Clinicopathologic characteristics and prognostic factors for HER2-positive patients with metastatic breast cancer in southern China. Arch Med Sci 2015;11:544-50. 
12. Marshall EM, Bertaut A, Desmoulins I, et al. Prognostic Factors of Survival among Women with Metastatic Breast Cancer and Impact of Primary or Secondary Nature of Disease on Survival: A French Population-Based Study. Breast J 2017;23:138-45.

13. Parkes A, Warneke CL, Clifton K, et al. Prognostic Factors in Patients with Metastatic Breast Cancer with Bone-Only Metastases. Oncologist 2018;23:1282-8.

14. Bringolf L, Pestalozzi B, Fink D, et al. Exploring prognostic factors for HER2-positive metastatic breast cancer: a retrospective cohort study in a major Swiss hospital. Swiss Med Wkly 2016;146:w14393.

15. Khanfir A, Lahiani F, Bouzguenda R, et al. Prognostic factors and survival in metastatic breast cancer: A single institution experience. Rep Pract Oncol Radiother 2013;18:127-32.

16. Coleman RE, Smith P, Rubens RD. Clinical course and prognostic factors following bone recurrence from breast cancer. Br J Cancer 1998;77:336-40.

17. Altundag K, Bondy ML, Mirza NQ, et al. Clinicopathologic characteristics and prognostic factors in 420 metastatic breast cancer patients with central nervous system metastasis. Cancer 2007;110:2640-7.

18. Largillier R, Ferrero JM, Doyen J, et al. Prognostic factors in 1,038 women with metastatic breast cancer. Ann Oncol 2008;19:2012-9.

19. Follana P, Barriere J, Chamorey E, et al. Prognostic factors in 401 elderly women with metastatic breast cancer. Oncology 2014;86:143-51.

20. Yamamura J, Masuda N, Yasojima H, et al. Clinicopathological Factors Related to the Prognosis of Metastatic Breast Cancer Patients after Development of Brain Metastasis. Breast Care (Basel) 2015;10:387-92.

21. Tazhibi M, Fayaz M, Mokarian F. Detection of prognostic factors in metastatic breast cancer. J Res Med Sci 2013;18:283-90.

22. Kawano A, Shimizu C, Hashimoto K, et al. Prognostic factors for stage IV hormone receptor-positive primary metastatic breast cancer. Breast Cancer 2013;20:145-51.

23. Ren Z, Li Y, Hameed O, et al. Prognostic factors in patients with metastatic breast cancer at the time of diagnosis. Pathol Res Pract 2014;210:301-6.

24. Chen MT, Sun HF, Zhao Y, et al. Comparison of patterns and prognosis among distant metastatic breast cancer patients by age groups: a SEER population-based analysis. Sci Rep 2017;7:9254.

25. Finn RS, Martin M, Rugo HS, et al. Palbociclib and Letrozole in Advanced Breast Cancer. N Engl J Med
2016;375:1925-36.

26. Hortobagyi GN, Stemmer SM, Burris HA, et al. Ribociclib as First-Line Therapy for HR-Positive, Advanced Breast Cancer. N Engl J Med 2016;375:1738-48.

27. Diéras V, Miles D, Verma S, et al. Trastuzumab emtansine versus capecitabine plus lapatinib in patients with previously treated HER2-positive advanced breast cancer (EMILIA): a descriptive analysis of final overall survival results from a randomised, open-label, phase 3 trial. Lancet Oncol 2017;18:732-42.

28. Robson M, Im SA, Senkus E, et al. Olaparib for Metastatic Breast Cancer in Patients with a Germline BRCA Mutation. N Engl J Med 2017;377:523-33.

29. Giarratano T, Miglietta F, Giorgi CA, et al. Exceptional and Durable Responses to TDM-1 After Trastuzumab Failure for Breast Cancer Skin Metastases: Potential Implications of an Immunological Sanctuary. Front Oncol 2018;8:581.

30. Litton JK, Rugo HS, Ettl J, et al. Talazoparib in Patients with Advanced Breast Cancer and a Germline BRCA Mutation. N Engl J Med 2018;379:753-63.

31. Ramos-Esquivel A, Hernandez-Steller H, Savard MF, et al. Cyclin-dependent kinase 4/6 inhibitors as firstline treatment for post-menopausal metastatic hormone receptor-positive breast cancer patients: a systematic review and meta-analysis of phase III randomized clinical trials. Breast Cancer 2018;25:479-88.

32. Ellegard S, Veenstra C, Perez-Tenorio G, et al. ERBB2 and PTPN2 gene copy numbers as prognostic factors in HER2-positive metastatic breast cancer treated with trastuzumab. Oncol Lett 2019;17:3371-81.

33. von Minckwitz G, Huang CS, Mano MS, et al. Trastuzumab Emtansine for Residual Invasive HER2Positive Breast Cancer. N Engl J Med 2019;380:617-28.

34. Piccart-Gebhart MJ, Procter M, Leyland-Jones B, et al. Trastuzumab after adjuvant chemotherapy in HER2positive breast cancer. N Engl J Med 2005;353:1659-72.

35. FDA. Abemaciclib. Available online: https:// www.accessdata.fda.gov/drugsatfda_docs/ label/2017/208716s000lbl.pdf

36. Fehm T, Sauerbrei W. Information from CTC measurements for metastatic breast cancer prognosiswe should do more than selecting an "optimal cut point". Breast Cancer Res Treat 2010;122:219-20.

37. Bulfoni M, Gerratana L, Del Ben F, et al. In patients with metastatic breast cancer the identification of circulating tumor cells in epithelial-to-mesenchymal transition is associated with a poor prognosis. Breast Cancer Res 
2016;18:30.

38. Mostert B, Sieuwerts AM, Kraan J, et al. Gene expression profiles in circulating tumor cells to predict prognosis in metastatic breast cancer patients. Ann Oncol 2015;26:510-6.

39. Ryberg M, Nielsen D, Osterlind K, et al. Prognostic factors and long-term survival in 585 patients with metastatic breast cancer treated with epirubicin-based chemotherapy. Ann Oncol 2001;12:81-7.

40. Chang J, Clark GM, Allred DC, et al. Survival of patients with metastatic breast carcinoma: importance of prognostic markers of the primary tumor. Cancer 2003;97:545-53.

41. Yamamoto N, Watanabe T, Katsumata N, et al. Construction and validation of a practical prognostic index for patients with metastatic breast cancer. J Clin Oncol 1998;16:2401-8.

42. Williams MR, Todd JH, Nicholson RI, et al. Survival patterns in hormone treated advanced breast cancer. Br J Surg 1986;73:752-5.

43. Andre F, Slimane K, Bachelot T, et al. Breast cancer with synchronous metastases: trends in survival during a 14year period. J Clin Oncol 2004;22:3302-8.

Cite this article as: $\mathrm{Hu} \mathrm{M}$, Shao $\mathrm{B}$, Ran $\mathrm{R}, \mathrm{Li} \mathrm{H}$. Prognostic factors for patients with metastatic breast cancer: a literature review. Transl Cancer Res 202 1;10(4):1644-1655. doi: 10.21037/ tcr-20-2119
44. Giordano SH, Buzdar AU, Smith TL, et al. Is breast cancer survival improving? Cancer 2004;100:44-52.

45. Lee YT. Breast carcinoma: pattern of metastasis at autopsy. J Surg Oncol 1983;23:175-80.

46. Lin NU, Bellon JR, Winer EP. CNS metastases in breast cancer. J Clin Oncol 2004;22:3608-17.

47. Maki DD, Grossman RI. Patterns of disease spread in metastatic breast carcinoma: influence of estrogen and progesterone receptor status. AJNR Am J Neuroradiol 2000;21:1064-6.

48. Lin NU, Winer EP. Brain metastases: the HER2 paradigm. Clin Cancer Res 2007;13:1648-55.

49. Slamon DJ, Godolphin W, Jones LA, et al. Studies of the HER-2/neu proto-oncogene in human breast and ovarian cancer. Science 1989;244:707-12.

50. Ferretti G, Felici A, Papaldo P, et al. HER2/neu role in breast cancer: from a prognostic foe to a predictive friend. Curr Opin Obstet Gynecol 2007;19:56-62.

51. Dawood S, Broglio K, Buzdar AU, et al. Prognosis of women with metastatic breast cancer by HER2 status and trastuzumab treatment: an institutional-based review. J Clin Oncol 2010;28:92-8. 